\title{
What is yet to be known about microbial-based systems for aquaculture?
}

Volume 5 Issue I - 2017

\section{Opinion}

The use of microbes as direct feed for fish and crustacean has emerged as a promissory alternative to improve aquaculture these organisms. Biofilm and biofloc have demonstrated positive effects on productive response of cultured fish and crustacean.

Biofilms are considered as aggregates of diverse microorganisms belonging to diverse domains that are embedded by exopolymeric substances and strongly attached to any surface, whereas microbial flocs (bioflocs) are formed by heterotrophic bacteria, phytoplankton, zooplankton and protozoa adhered to a floating surface. ${ }^{1,2}$ These have proven to serve not only as direct food source for the cultured organisms, but also as promoters of a short food chain within culture units, causing the recirculation of nutrients and promoting proliferation of bacteria that could inhibit the reproduction of pathogenic specimens. ${ }^{3}$ In addition, microbial-based systems allow to minimize water exchange in aquaculture systems through maintaining adequate water quality within the culture unit, while producing lowcost edible biomass rich in protein and other nutrients contributing to the sustainability of aquaculture. ${ }^{4}$ Several studies have demonstrated the nutritional quality of these microbial consortia, and have addressed standardization of protocols to achieve optimal biofilm formation or flocculation, nutritional composition, and etcetera. ${ }^{4}$ However, apart to know more about the technical management of these strategies, one of the main challenges of this discipline is to know the biology of these conglomerates as well as the identification and characterization of microorganisms yielding biofilm and biofloc that provide benefits to aquaculture systems or that can be used as inoculum. This is difficult, because at first it is necessary to know the microbial diversity thriving in these complex conglomerates.

Information about the microbial diversity detected in biofilm or biofloc used in aquaculture is usually reported at superficial taxonomic levels and only a small fraction of the total diversity is studied. However, significant discussions and conclusions regarding the biology of these conglomerates can be only achieved when their microbes can be identified at higher taxonomic levels. This is not a readily task, because most of the prokaryotes inhabiting any environment are not culturable or difficult to culture. Fortunately the overcome of culture-independent techniques such as high throughput sequencing has provided a way for the high resolution study of these microbes.

Targeted metagenomics considering single genes that could serve as biomarkers for taxonomic identity may reveal a wide spectrum of microbes contained in biofilm and biofloc. For instance, MartínezCórdova et al. ${ }^{1}$ studied the diversity of bacteria thriving into a phototrophic biofilm using the variable regions 3 and 4 of the $16 \mathrm{~S}$ rRNA gene. The authors reported the presence of tens of bacteria species and hypothesized the occurrence of a complex interaction network functioning to maintain the equilibrium of the microbial community. Regarding biofloc used in aquaculture, Zhao et al. ${ }^{5}$ studied the bacteria contained into biofloc used for penaeid shrimp.

\author{
Marcel Mart, ' Vargas-Albores F,' ${ }^{2}$ Mart M² $^{2}$ \\ Centro de Investigaciones Biol \\ ${ }^{2}$ Centro de Investigaci
}

Correspondence: Marcel Martínez-Porchas, Centro de Investigación en Alimentación y Desarrollo,AC, Coordinación de Tecnología de Alimentos de Origen Animal, Mexico Email marcel@ciad.mx

Received: January 09, 2017 | Published: January II, 2017

The authors used a culture-independent technique considering the same variable regions of the $16 \mathrm{~S}$ rRNA gene, but using a method with lower resolution (DGGE) and reported the most abundant genus which resulted to be Bacillus. However, these constitute first approaches to know some of the bacteria thriving in these conglomerates.

The next step is to answer the following question, what are they doing or are they able to do? This can be also addressed by high throughput sequencing, particularly through shotgun sequencing. This last method produces several amounts of noisy data that may have nonsense at simple sight; however, current bioinformatics tools as well as computer capacities can be used for the reconstruction of genomes and transcriptomes from the environment, allowing to analyze the community structure and metabolism of these conglomerates. ${ }^{6}$ Other questions could be answered with the correct interpretation of these data; for instance: which environmental conditions trigger the growth and proliferation of certain species?, how do different carbon or nitrogen sources affect diversity and metabolism of these communities?, how do these conglomerates inhibit the growth of pathogens and recirculate nutrients?, how do the production and consumption of these conglomerates influence pond and shrimp intestinal microbiota?, are these communities transfer inductors of antibiotic resistance genes?, and etcetera.

There is still much information waiting to be revealed about these conglomerates, but many of the questions will be responded by studying the biology of these communities, including structure and functions. Finally, other domains apart of bacteria, such as archaea, eukarya, rhodophyta, fungi and etcetera have to be considered to extend the research panorama. Part of the future of aquaculture lies in the proper use of microbes to feed cultured organisms, but there is still much to know about the subject.

\section{Acknowledgments}

None. 


\section{Conflicts of interest}

None.

\section{References}

1. Martínez-Córdova L, Martínez-Porchas M, Porchas-Cornejo M, et al. Bacterial diversity studied by next-generation sequencing in a mature phototrophic Navicula sp-based biofilm promoted into a shrimp culture system. Aquaculture Research. 2016.

2. Najdegerami EH, Bakhshi F, Lakani FB. Effects of biofloc on growth performance, digestive enzyme ctivities and liver histology of common carp (Cyprinus carpio L.) fingerlings in zero-water exchange system. Fish physiol Biochem. 2016;42(2):457-465.
3. Martínez-Córdova L, Martínez-Porchas M, Emerenciano M, et al. From microbes to fish the next revolution in food production. Critical Reviews in Biotechnology. 2016;10:1-9.

4. Crab R, Defoirdt T, Bossier P, et al. Biofloc technology in aquaculture: beneficial effects and future challenges. Aquaculture. 2012;356357:351-356

5. Zhao P, Huang J, Wang X-H, et al. The application of bioflocs technology in high-intensive, zero exchange farming systems of Marsupenaeus japonicus. Aquaculture. 2012;354-355:97-106.

6. Martinez-Porchas M, Vargas-Albores F. Microbial metagenomics in aquaculture: a potential tool for a deeper insight into the activity. Reviews in Aquaculture In press. 2015. 